\title{
Realization of Boolean Control Networks *
}

\author{
Daizhan Cheng*, Zhiqiang Li, Hongsheng Qi \\ Key Laboratory of Systems and Control, Academy of Mathematics and Systems Science, \\ Chinese Academy of Sciences, Beijing 100190, P.R.China
}

\begin{abstract}
Based on the linear expression of the dynamics of Boolean networks, the coordinate transformation of Boolean variables is defined. It follows that the state space coordinate transformation for the dynamics of Boolean networks is revealed. Using it, the invariant subspace for a Boolean control network is defined. Then the structure of a Boolean control network is analyzed, and the controllable and observable normal forms and the Kalman decomposition form are presented. Finally the realization problem, including minimum realization, of Boolean control networks is investigated.
\end{abstract}

Key words: Boolean control network; coordinate transformation; normal form; Kalman decomposition; realization.

\section{Introduction}

In recent years gene control networks have caused an emergence of interest in the quantitative description of gene regulation (Davidson et al., 2002; Albert and Othmer, 2003). The Boolean network, first introduced by Kauffman (Kauffman, 1969), has been proved to be quite useful in modeling and quantitative description of cell regulation (Kauffman, 1969; Huang and Ingber, 2000; Huang, 2002; Farrow et al., 2004).

Recently, we use a new matrix product, denoted by " $\ltimes$ " and called the semi-tensor product (Cheng, 2007), to convert a logical function into an algebraic function. Furthermore, the logical dynamics of a Boolean network is converted into a standard discrete-time dynamics. Based on this, a new technique has been developed for analyzing and synthesizing Boolean (control) networks (Cheng and Qi, 2009b; Cheng, 2009; Cheng and Qi, 2009a).

The purpose of this paper is to use this new technique to analyze the input-output relations of Boolean control networks. First we consider the controllable and observable normal forms. Then the Kalman decomposition is

\footnotetext{
* Supported partly by NNSF 60674022, 60736022, and 60821091 of China.

* Corresponding author. Tel. +86-10-6265 1445. Fax +8610-6258 7343 .

Email addresses: dcheng@iss.ac.cn (Daizhan Cheng),

lizhiqiang@amss.ac.cn (Zhiqiang Li),

qihongsh@amss.ac.cn (Hongsheng Qi).
}

obtained. Finally, the realization, particularly, minimum realization, of Boolean control networks is investigated.

For this purpose, two new important concepts have been introduced: (a) the coordinate transformation of Boolean (control) networks; (b) regular subspace of the state space.

The paper is organized as follows. Section 2 provides some preliminaries. Section 3 investigates when a mapping on two sets of $n$ independent logical variables can be a coordinate transformation, and how to construct the inverse mapping of a given coordinate transformation. Section 4 discusses the regular subspace of the state space. Section 5 considers the state space coordinate transformation of Boolean networks and Boolean control networks, which provides a tool for normal forms of a Boolean control network. Then in Section 6 the controllable and observable normal forms are obtained. Moreover, using the normal forms the Kalman decomposition form is also obtained. Section 7 considers the equivalent realization and the minimum realization of a Boolean control network. Section 8 is a brief conclusion.

\section{Preliminaries}

\subsection{Matrix Expression of Logic}

A logical variable takes value from $\mathcal{D}=\{1,0\}$, where $1 \sim$ $T$ and $0 \sim F$ represent "True" and "False" respectively. To use matrix expression, we use two vectors to represent 
these two logical values as

$$
T \sim 1 \sim \delta_{2}^{1}, \quad F \sim 0 \sim \delta_{2}^{2}
$$

where $\delta_{n}^{k}$ denotes the $k$ th column of the identity matrix $I_{n}$. We set

$$
\Delta_{n}:=\left\{\delta_{n}^{k} \mid 1 \leq k \leq n\right\} .
$$

For notational ease, $\Delta_{2}:=\Delta$. Then $\Delta \sim \mathcal{D}$.

An $n \times t$ matrix $M$ is called a logical matrix if

$$
M=\left[\begin{array}{llll}
\delta_{n}^{i_{1}} & \delta_{n}^{i_{2}} & \cdots & \delta_{n}^{i_{t}}
\end{array}\right]
$$

The set of $n \times t$ logical matrices is denoted by $\mathcal{L}_{n \times t}$. For compactness, we briefly denote above $M$ as

$$
M=\delta_{n}\left[i_{1}, i_{2}, \cdots, i_{t}\right]
$$

In vector form we have the following fundamental result (Cheng, 2007).

Theorem 1. Let $f\left(x_{1}, x_{2}, \cdots, x_{s}\right)$ be a logical function. Then there exists unique $M_{f} \in \mathcal{L}_{2 \times 2^{s}}$, called the structure matrix of $f$, such that

$$
f\left(x_{1}, x_{2}, \cdots, x_{s}\right)=M_{f} \ltimes_{i=1}^{s} x_{i}, \quad x_{i} \in \Delta .
$$

In Table 1 we list the structure matrices for some basic logical operators (LO) (Negation: $\neg$; Conjunction: $\wedge$; Disjunction: $\vee$; Conditional: $\rightarrow$; Biconditional: $\leftrightarrow$; Exclusive Or: $\bar{V}$ (Rade and Westergren, 1998)), which are used in what follows.

Table 1

Structure Matrices of Some Basic Logical Operators

\begin{tabular}{|c|c||c|c|}
\hline LO & Structure Matrix & LO & Structure Matrix \\
\hline$\neg$ & $M_{n}=\delta_{2}\left[\begin{array}{lll}2 & 1\end{array}\right]$ & $\vee$ & $M_{d}=\delta_{2}\left[\begin{array}{llll}1 & 1 & 1 & 2\end{array}\right]$ \\
\hline$\rightarrow$ & $M_{i}=\delta_{2}\left[\begin{array}{llll}1 & 2 & 1 & 1\end{array}\right]$ & $\leftrightarrow$ & $M_{e}=\delta_{2}\left[\begin{array}{llll}1 & 2 & 2 & 1\end{array}\right]$ \\
\hline$\wedge$ & $M_{c}=\delta_{2}\left[\begin{array}{llll}1 & 2 & 2 & 2\end{array}\right]$ & $\bar{\nabla}$ & $M_{p}=\delta_{2}\left[\begin{array}{llll}2 & 1 & 1 & 2\end{array}\right]$ \\
\hline
\end{tabular}

Finally, we define the swap matrix (Cheng, 2007): An $m n \times m n$ matrix $W_{[m, n]}$ is called a swap matrix, if

$$
W_{[m, n]} X Y=Y X, \quad \forall X \in \mathbb{R}^{m}, \forall Y \in \mathbb{R}^{n} .
$$

$W_{[m, n]}$ uniquely exists.

\subsection{Boolean Control Networks}

A Boolean control network is a Boolean network with additional inputs and outputs. Its dynamics can be expressed as follows (Akutsu et al., 2007; Cheng, 2009;
Cheng and Qi, 2009a)

$$
\left\{\begin{array}{l}
x_{1}(t+1)=f_{1}\left(x_{1}(t), \cdots, x_{n}(t), u_{1}(t), \cdots, u_{m}(t)\right) \\
x_{2}(t+1)=f_{2}\left(x_{1}(t), \cdots, x_{n}(t), u_{1}(t), \cdots, u_{m}(t)\right) \\
\vdots \\
x_{n}(t+1)=f_{n}\left(x_{1}(t), \cdots, x_{n}(t), u_{1}(t), \cdots, u_{m}(t)\right), \\
y_{i}(t)=h_{i}\left(x_{1}(t), \cdots, x_{n}(t)\right), \quad i=1, \cdots, p,
\end{array}\right.
$$

where $x_{i}(t) \in \Delta$ are logical variables, $f_{i}, i=1, \cdots, n$, and $h_{i}, i=1, \cdots, p$ are logical functions, $u_{i}(t) \in \Delta$, $i=1, \cdots, m$ are controls, $y_{i}(t) \in \Delta, i=1, \cdots, p$ are outputs.

We use an example to depict it.

Example 2. Fig. 1 consists of a Boolean network with four nodes $x_{1}, x_{2}, x_{3}, x_{4}$ as its state variables. Moreover, we have two inputs $u_{1}, u_{2}$ acting on the network and one output $y$ as a logical function of state variables.

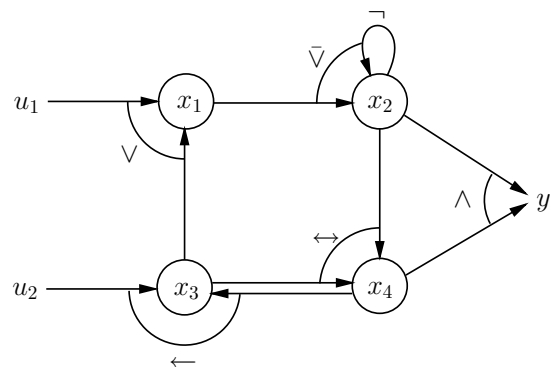

Fig. 1. A Boolean control network

Its dynamics is described as

$$
\left\{\begin{array}{l}
x_{1}(t+1)=x_{3}(t) \vee u_{1}(t) \\
x_{2}(t+1)=x_{1}(t) \bar{\vee}\left(\neg x_{2}(t)\right) \\
x_{3}(t+1)=x_{4}(t) \rightarrow u_{2}(t) \\
x_{4}(t+1)=x_{2}(t) \leftrightarrow x_{3}(t) \\
y(t)=x_{2}(t) \wedge x_{4}(t)
\end{array}\right.
$$

\subsection{A Motivating Example}

Consider a control system, it may be reasonable to say that the most important characteristic of the system is its input-output mapping. Roughly speaking, if two systems realize a same input-output mapping, they are said to be equivalent. Particularly, if there is a state space coordinate transformation, which converts one system into the other, then we can simply say that they are the same. 
Now a natural question is: are there any coordinate transformations, which express the same system into different forms? More general, is it possible that two different Boolean control systems with different sizes realize a same input-output mapping? The answer is "yes". We give a heuristic example.

Example 3. Consider the following two systems

$$
\Sigma_{1}:\left\{\begin{aligned}
x_{1}(t+1)= & u \leftrightarrow \neg\left(x_{1}(t) \rightarrow x_{2}(t)\right) \\
x_{2}(t+1)= & \left(u \wedge\left(\neg x_{1}(t) \wedge x_{2}(t)\right)\right) \vee \\
& \left(\neg u \wedge \neg\left(x_{1}(t) \rightarrow x_{1}(t)\right)\right) \\
y(t)=x_{1}(t) & \leftrightarrow x_{2}(t),
\end{aligned}\right.
$$

and

$$
\Sigma_{2}: \quad\left\{\begin{array}{l}
z_{1}(t+1)=z_{1}(t) \wedge u \\
z_{2}(t+1)=\left(z_{1}(t) \vee z_{2}(t)\right) \leftrightarrow u \\
y(t)=z_{1}(t)
\end{array}\right.
$$

It is not difficult to verify that as the initial values satisfy

$$
\left\{\begin{array}{l}
z_{1}(0)=x_{1}(0) \leftrightarrow x_{2}(0) \\
z_{2}(0)=\neg x_{1}(0)
\end{array}\right.
$$

the input-output mappings of $\Sigma_{1}$ and $\Sigma_{2}$ are exactly the same. So a natural guess is: $\Sigma_{2}$ is obtained from $\Sigma_{1}$ via a "coordinate transformation"

$$
\left\{\begin{array}{l}
z_{1}=x_{1} \leftrightarrow x_{2} \\
z_{2}=\neg x_{1}
\end{array}\right.
$$

In fact, this is true, and you can verify this later after we give a rigorous definition about coordinate change.

Moreover, we can also see that in fact the output of $\Sigma_{2}$ depends only on $z_{1}$ and $z_{1}$ is independent of $z_{2}$. So $z_{2}$ is a redundant state variable regarding the realization of the input-output mapping. We, therefore, can remove it to obtain the following

$$
\Sigma_{3}:\left\{\begin{array}{l}
z(t+1)=z(t) \wedge u \\
y(t)=z(t)
\end{array}\right.
$$

Now as long as the initial conditions of $\Sigma_{1}$ and $\Sigma_{3}$ satisfy the condition that $z(0)=x_{1}(0) \leftrightarrow x_{2}(0)$, they realize the same input-output mapping.

From this example one sees that similar to conventional (qualitative) control systems, to consider the realization of a logical control network the coordinate transformation is necessary. It is very likely that "minimum realization" can be found under a suitable coordinate frame.

\section{Coordinate Transformation on $\mathcal{D}^{n}$}

Assume that $\left\{x_{1}, x_{2}, \cdots, x_{n}\right\}$ is a set of independent logical variables, and there is another set of logical variables $\left\{y_{1}, y_{2}, \cdots, y_{n}\right\}$. Moreover, $y_{i}$ are logical functions of $x_{i}$, $i=1, \cdots, n$, denoted as

$$
\Psi:\left\{\begin{aligned}
y_{1} & =q_{1}\left(x_{1}, x_{2}, \cdots, x_{n}\right), \\
y_{2} & =q_{2}\left(x_{1}, x_{2}, \cdots, x_{n}\right), \\
\vdots & \\
y_{n} & =q_{n}\left(x_{1}, x_{2}, \cdots, x_{n}\right) .
\end{aligned}\right.
$$

Definition 4. The mapping $\Psi: \mathcal{D}^{n} \mapsto \mathcal{D}^{n}$ is called a logical coordinate transformation (briefly, coordinate change), if it is one-to-one and onto.

Using vector form, we denote $y=\ltimes_{i=1}^{n} y_{i} \in \Delta_{2^{n}}$ and $x=\ltimes_{i=1}^{n} x_{i} \in \Delta_{2^{n}}$. Then (9) can be expressed in an algebraic form as

$$
y=T_{\Psi} x
$$

where $T_{\Psi} \in \mathcal{L}_{2^{n} \times 2^{n}}$ is called the transfer matrix of the mapping $\Psi$. The following proposition is obvious.

Proposition 5. Equation (9) forms a logical coordinate change, iff its transfer matrix $T_{\Psi}$ is nonsingular. Moreover, since $T_{\Psi} \in \mathcal{L}_{2^{n} \times 2^{n}}$, nonsingularity implies that

$$
T_{\Psi}^{-1}=T_{\Psi}^{T}
$$

For the applications in what follows, we have to construct the inverse logical functions, denoted by

$$
\Psi^{-1}: x_{i}=p_{i}\left(y_{1}, \cdots, y_{n}\right), \quad i=1, \cdots, n,
$$

from its transition matrix $T_{\Psi}^{-1}=T_{\Psi}^{T}$. We recall how to construct the inverse mapping $\Psi^{-1}$ (Cheng and Qi, 2009a). Define a set of matrices, called the retrievers, as

$$
\begin{aligned}
& S_{1}^{n}=\delta_{2}[\underbrace{1, \cdots, 1}_{2^{n-1}}, \underbrace{2, \cdots, 2}_{2^{n-1}}] ; \\
& S_{2}^{n}=\delta_{2}[\underbrace{1, \cdots, 1}_{2^{n-2}}, \underbrace{2, \cdots, 2}_{2^{n-2}}, \underbrace{1, \cdots, 1}_{2^{n-2}}, \underbrace{2, \cdots, 2}_{2^{n-2}}] ; \\
& \vdots \\
& S_{n}^{n}=\delta_{2}[1,2,1,2, \cdots, 1,2] .
\end{aligned}
$$

Then the structure matrix of $p_{i}$, denoted by $P_{i}$, can be obtained as

$$
P_{i}=S_{i}^{n} T_{\Psi}^{T}, \quad i=1,2, \cdots, n .
$$


To get the logical equation $p_{i}$ from $P_{i}$, Cheng and Qi (2009a) provides the following method: split $P_{i}$ into two equal part as

$$
P_{i}=\left[P_{i}^{1} P_{i}^{2}\right]
$$

Then $p_{i}$ can be expressed as

$$
\begin{aligned}
p_{i}\left(x_{1}, \cdots, x_{n}\right)= & \left(x_{1} \wedge p_{i}^{1}\left(x_{2}, \cdots, x_{n}\right)\right) \vee \\
& \left(\neg x_{1} \wedge p_{i}^{2}\left(x_{2}, \cdots, x_{n}\right)\right),
\end{aligned}
$$

where $p_{i}^{1}$ and $p_{i}^{2}$ have $P_{i}^{1}$ and $P_{i}^{2}$ as their structure matrices respectively. Continuing this process, a disjunctive normal form of $p_{i}$ is produced.

We give an example to depict this.

Example 6. Consider a set of mappings

$$
\left\{\begin{array}{l}
y_{1}=\neg x_{2} \\
y_{2}=x_{1} \leftrightarrow x_{2} \\
y_{3}=\neg x_{3} .
\end{array}\right.
$$

Using the algebraic expression, we have

$$
\left\{\begin{array}{l}
y_{1}=M_{n} x_{2} \\
y_{2}=M_{e} x_{1} x_{2} \\
y_{3}=M_{n} x_{3} .
\end{array}\right.
$$

Set $x=x_{1} x_{2} x_{3}, y=y_{1} y_{2} y_{3}$. Then

$$
\begin{aligned}
y & =y_{1} y_{2} y_{3} \\
& =M_{n} x_{2} M_{e} x_{1} x_{2} M_{n} x_{3} \\
& =M_{n}\left(I_{2} \otimes M_{e}\right) W_{[2]} x_{1} x_{2}^{2} M_{n} x_{3} \\
& =M_{n}\left(I_{2} \otimes M_{e}\right) W_{[2]}\left(I_{2} \otimes M_{r}\right) x_{1} x_{2} M_{n} x_{3} \\
& =M_{n}\left(I_{2} \otimes M_{e}\right) W_{[2]}\left(I_{2} \otimes M_{r}\right)\left(I_{4} \otimes M_{n}\right) x_{1} x_{2} x_{3} \\
& :=T x .
\end{aligned}
$$

Then $T \in \mathcal{L}_{8 \times 8}$ is

$$
\begin{aligned}
T & =M_{n}\left(I_{2} \otimes M_{e}\right) W_{[2]}\left(I_{2} \otimes M_{r}\right)\left(I_{4} \otimes M_{n}\right) \\
& =\delta_{8}[6,5,4,3,8,7,2,1] .
\end{aligned}
$$

Since $T$ is nonsingular, (16) is a logical coordinate transformation.

To get the inverse transformation, we have

$$
x=T^{-1} y=T^{T} y .
$$

Then

$$
\begin{aligned}
& x_{1}=S_{1}^{3} T^{T} y:=M_{1} y=\delta_{2}\left[\begin{array}{llllllll}
2 & 2 & 1 & 1 & 1 & 1 & 2 & 2
\end{array}\right] y_{1} y_{2} y_{3} \\
& x_{2}=S_{2}^{3} T^{T} y:=M_{2} y=\delta_{2}\left[\begin{array}{lllllllll}
2 & 2 & 2 & 2 & 1 & 1 & 1 & 1
\end{array}\right] y_{1} y_{2} y_{3} \\
& x_{3}=S_{3}^{3} T^{T} y:=M_{3} y=\delta_{2}\left[\begin{array}{lllllllll}
2 & 1 & 2 & 1 & 2 & 1 & 2 & 1
\end{array}\right] y_{1} y_{2} y_{3} .
\end{aligned}
$$

Using the standard process to convert them back to logical form, we denote

$$
x_{1}=\left[y_{1} \wedge g_{1}^{1}\left(y_{2}, y_{3}\right)\right] \vee\left[\neg y_{1} \wedge g_{1}^{2}\left(y_{2}, y_{3}\right)\right],
$$

Then

$$
\begin{aligned}
& g_{1}^{1}\left(y_{2}, y_{3}\right)=M_{1}^{1} y_{2} y_{3}=\delta_{2}\left[\begin{array}{llll}
2 & 2 & 1 & 1
\end{array}\right] y_{2} y_{3}=\neg y_{2} \text {; } \\
& g_{1}^{2}\left(y_{2}, y_{3}\right)=M_{1}^{2} y_{2} y_{3}=\delta_{2}\left[\begin{array}{llll}
1 & 1 & 2 & 1
\end{array}\right] y_{2} y_{3}=y_{2} \text {. }
\end{aligned}
$$

Hence we have

$$
x_{1}=\left(y_{1} \wedge \neg y_{2}\right) \vee\left(\neg y_{1} \wedge y_{2}\right)=y_{1} \bar{\vee} y_{2} .
$$

Similarly, we can get $x_{2}$ and $x_{3}$ as

$$
x_{2}=\neg y_{1} ; \quad x_{3}=\neg y_{3} .
$$

\section{Regular Subspace}

Definition 7. Let $\left\{x_{1}, \cdots, x_{k}\right\}$ be a set of logical variables. The logical space generated by $\left\{x_{1}, \cdots, x_{k}\right\}$, denoted by $\mathcal{S}=F_{\ell}\left\{x_{1}, \cdots, x_{k}\right\}$, is the set of logical functions of $\left\{x_{1}, \cdots, x_{k}\right\}$.

Consider system (2). $x_{1}, \cdots, x_{n}$ are called the state variables. The state space of (2) is defined as

$$
\mathcal{U}=F_{\ell}\left\{x_{1}, \cdots, x_{n}\right\}
$$

Definition 8. (1) Let $\left\{y_{1}, \cdots, y_{r}\right\}(r \leq n)$ be a set of logical variables in $\mathcal{U}$. $\left\{y_{1}, \cdots, y_{r}\right\}$ is called a regular sub-basis of $\mathcal{U}$, if we can find $y_{r+1}, \cdots, y_{n}$, such that $y_{1}, \cdots, y_{n}$ is a coordinate change of $x$.

(2) $\mathcal{S} \subset \mathcal{U}$ is called a regular subspace of $\mathcal{U}$ if there exists a regular sub-basis $\left\{y_{1}, \cdots, y_{r}\right\}$, such that $\mathcal{S}=F_{\ell}\left\{y_{1}, \cdots, y_{r}\right\}$.

Given a set of functions $y_{i}$ as

$$
y_{i}=g_{i}\left(x_{1}, \cdots, x_{n}\right), \quad i=1, \cdots, r,
$$

we would like to know when it is a regular sub-basis. Set $y=\ltimes_{i=1}^{r} y_{i}$ and $x=\ltimes_{i=1}^{n} x_{i}$. From (21) we can easily get its algebraic form as

$$
y=L x:=\left[\begin{array}{cccc}
\ell_{11} & \ell_{12} & \cdots & \ell_{1,2^{n}} \\
\vdots & & & \\
\ell_{2^{r}, 1} & \ell_{2^{r}, 2} & \cdots & \ell_{2^{r}, 2^{n}}
\end{array}\right] x .
$$

Proposition 9. Assume that the structure matrix of $g_{i}$ is

$$
M_{i}=\left[\xi_{1}^{i} \xi_{2}^{i} \cdots \xi_{2^{n}}^{i}\right], \quad i=1, \cdots, r .
$$


Then

$$
L=\left[\begin{array}{llll}
\ell_{1} & \ell_{2} & \cdots & \ell_{2^{n}}
\end{array}\right]
$$

where

$$
\ell_{k}=\ltimes_{i=1}^{r} \xi_{k}^{i}, \quad k=1, \cdots, 2^{n} .
$$

PROOF. Assume $x_{1}=x_{2}=\cdots=x_{n}=\delta_{2}^{1} \sim 1$. By the construction of structure matrix it is easily seen that $y_{i}=\xi_{1}^{i}, i=1, \cdots, r$. Hence $y=\ltimes_{i=1}^{r} \xi_{1}^{i}$. Similarly, let $x_{i}=\alpha_{i} \in\{0,1\}, i=1, \cdots, n$, and set $k=2^{n}-\left[\alpha_{1} \times\right.$ $\left.2^{n-1}+\alpha_{2} \times 2^{n-2}+\cdots+\alpha_{n}\right]$. Then $y_{i}=\xi_{k}^{i}, i=1, \cdots, r$. Hence $y=\ltimes_{i=1}^{r} \xi_{k}^{i}$.

The following corollary is easily verifiable.

Corollary 10. Assume that $y_{1}, \cdots, y_{p}$ and $z_{1}, \cdots, z_{q}$ are two sets of logical functions of $x_{1}, \cdots, x_{n}$. Denote $y=\ltimes_{i=1}^{p} y_{i}, z=\ltimes_{i=1}^{q} z_{i}, w=y z$, and $x=\ltimes_{i=1}^{n} x_{i}$. Moreover,

$$
y=M x, \quad z=N x, \quad w=L x,
$$

where $M, N$, and L are $2^{p} \times 2^{n}, 2^{q} \times 2^{n}$, and $2^{p+q} \times 2^{n}$ logical matrices respectively. Denote by $M^{i}$ the ith column of $M$ etc. Then we have

$$
L^{i}=M^{i} N^{i}, \quad i=1, \cdots, 2^{n} .
$$

The following theorem shows when $\left\{y_{1}, \cdots, y_{r}\right\}$ is a regular sub-basis.

Theorem 11. Assume that there is a set of logical variables $y_{1}, \cdots, y_{r}(r \leq n)$ satisfying (22). It is a regular sub-basis, iff the corresponding coefficient matrix $L$ satisfies

$$
\sum_{i=1}^{2^{n}} \ell_{k, i}=2^{n-r}, \quad k=1,2, \cdots, 2^{r}
$$

PROOF. (Sufficiency) Note that condition (24) means there are $2^{n-r}$ different $x$ which makes $y=\delta_{2^{r}}^{k}, k=$ $1,2, \cdots, 2^{r}$. Now we can choose $y_{r+1}$ as follows. Set

$$
S_{r}^{k}=\left\{x \mid L x=\delta_{2^{r}}^{k}\right\}, \quad k=1,2, \cdots, 2^{r} .
$$

Then the cardinal number $\left|S_{r}^{k}\right|=2^{n-r}$. For half of the elements of $S_{r}^{k}$, define $y_{r+1}=0$, and for the other half, set $y_{r+1}=1$. Then it is easy to see that for $\tilde{y}=\ltimes_{i=1}^{r+1} y_{i}$ the corresponding $\tilde{L}$ satisfies (24) with $r$ being replaced by $r+1$.
Continuing this process till $r=n$. Then (24) becomes

$$
\sum_{i=1}^{2^{n}} \ell_{k, i}=1, \quad k=1,2, \cdots, 2^{n}
$$

(25) means the corresponding $L$ contains all the columns of $I_{2^{n}}$, i.e., it is obtained from $I_{2^{n}}$ via a column permutation. It is, hence, a coordinate change.

(Necessity) Note that using the swap matrix, it is easy to see that the order of $y_{i}$ does not affect the property of (24). First, we claim that if $\left\{y_{i} \mid i=1, \cdots, k\right\}$ satisfies (24), then any of its subset $\left\{y_{i_{t}}\right\} \subset\left\{y_{i} \mid i=1, \cdots, k\right\}$ also satisfies (24). Since the order does not affect this property, it is enough to show that a $k-1$ subset $\left\{y_{i} \mid i=\right.$ $2, \cdots, k\}$ is a proper sub-basis, because from $k-1$ we can go to $k-2$ and so on. Assume that $y^{2}=\ltimes_{i=2}^{k} y_{i}=Q x$, and $y_{1}=P x$. Using Corollary 10, we have

$$
L^{i}=P^{i} Q^{i}, \quad i=1, \cdots, 2^{n} .
$$

Next, we split $L$ into two blocks with equal size as

$$
L=\left[\begin{array}{l}
L_{1} \\
L_{2}
\end{array}\right]
$$

Note that either $P^{i}=\delta_{2}^{1}$ or $P^{i}=\delta_{2}^{2}$. Using this fact to (26), one sees easily that either $L^{i}=\left[\begin{array}{c}Q^{i} \\ 0\end{array}\right]\left(\right.$ as $\left.P^{i}=\delta_{2}^{1}\right)$ or $L^{i}=\left[\begin{array}{c}0 \\ Q^{i}\end{array}\right]\left(\right.$ as $\left.P^{i}=\delta_{2}^{2}\right)$. Hence, $Q^{i}=L_{1}^{i}+L_{2}^{i}$. It follows that

$$
Q=L_{1}+L_{2}
$$

Since $L$ satisfies (24), (27) assures that $Q$ satisfies (24) too.

Now since $\left\{y_{i} \mid i=1, \cdots, k\right\}$ is a proper sub-basis, so there exists $\left\{y_{i} \mid i=k+1, \cdots, n\right\}$ such that $\left\{y_{i} \mid i=\right.$ $1, \cdots, n\}$ is a coordinate transformation of $x$, it satisfies (24). (Precisely, it satisfies (25) with row sum equal to 1.) According to the claim, the subset $\left\{y_{i} \mid i=1, \cdots, k\right\}$ also satisfies (24).

We give a simple example to explain this.

Example 12. Let $x_{1}, x_{2}$ be a basis. (i) Consider $y=$ $x_{1} \wedge x_{2}$. Since

$$
M_{c}=\left[\begin{array}{llll}
1 & 0 & 0 & 0 \\
0 & 1 & 1 & 1
\end{array}\right]
$$


$\sum_{i=1}^{4} \ell_{1 i}=1$, and $\sum_{i=1}^{4} \ell_{2 i}=3$. Hence $y$ cannot be a regular sub-basis.

(ii) Consider $z=x_{1} \leftrightarrow x_{2}$. Since

$$
M_{e}=\left[\begin{array}{llll}
1 & 0 & 0 & 1 \\
0 & 1 & 1 & 0
\end{array}\right] \text {, }
$$

$\sum_{i=1}^{4} \ell_{1 i}=2$, and $\sum_{i=1}^{4} \ell_{2 i}=2$. Hence $z$ is a regular subbasis.

The constructive proof of the sufficiency of Theorem 11 provides a way to construct a basis from a regular subbasis. Since $M_{e}=\delta_{2}[1,2,2,1]$, we need to find a $y$ such that $M_{y}$ has half 1 and half 2 in the position of 1 (or 2 ) of $M_{e}$. So, $M_{y}$ should be one of the followings: $\delta_{2}[1,1,2,2]$, $\delta_{2}[1,2,1,2], \delta_{2}[2,1,2,1], \delta_{2}[2,2,1,1]$. That is, $y=x_{1}$, $y=x_{2}, y=\neg x_{2}, y=\neg x_{1}$, correspondingly. Then $\{z, y\}$ becomes a coordinate transformation.

Next, we consider a set of nested regular sub-bases.

Theorem 13. Let $y_{1}, \cdots, y_{s}$ and $z_{1}, \cdots, z_{t}$ be two regular sub-bases of $x_{1}, \cdots, x_{n}$. Assume

$$
y_{i} \in F_{\ell}\left\{z_{1}, \cdots, z_{t}\right\}, \quad i=1, \cdots, s .
$$

Then $y_{1}, \cdots, y_{s}$ is also a regular sub-basis of $z_{1}, \cdots, z_{t}$.

PROOF. Choosing $z_{t+1}, \cdots, z_{n}$, such that $\tilde{z}=$ $\ltimes_{i=t+1}^{n} z_{i} \ltimes_{i=1}^{t} z_{i}$ is a coordinate transformation of $x$. It is easy to check that if $y=\ltimes_{i=1}^{s} y_{i}$ is a regular sub-basis with respect to $x=\ltimes_{i=1}^{n} x_{i}$, it is also a regular sub-basis with respect to $\tilde{z}$, i.e., "regularity" is independent of a particular choice of the coordinates. So we have

$$
y=H \tilde{z}:=\left[H_{1}, H_{2}\right] \tilde{z},
$$

where $H$ satisfies (24) and $H_{1}$ and $H_{2}$ are two equal size blocks of $H$. Setting $z_{t+1}=\delta_{2}^{1}$ we have $H_{1} z^{\prime}$, and setting $z_{t+1}=\delta_{2}^{2}$ we have $H_{2} z^{\prime}$, where $z^{\prime}=\ltimes_{i=t+2}^{n} z_{i} \ltimes_{i=1}^{t} z_{i}$. Now since $y$ is independent of $z_{t+1}$, we conclude that $H_{1}=H_{2}$. Removing the fabricated variable $z_{t+1}$ from (28) yields

$$
y=\left[H_{1}\right] z^{\prime}
$$

Since $H_{1}=H_{2}$, one sees that $H_{1}$ satisfies (24). Continuing this procedure, we can finally have

$$
y=H_{0} z,
$$

where $z=\ltimes_{i=1}^{s} z_{i}$, and $H_{0}$ satisfies (24). The conclusion follows from Theorem 11 .
Using Theorem 13, we can construct a universal coordinate frame for a set of nested regular sub-bases. The following corollary is obvious.

Corollary 14. Let $\left\{z_{1}^{i}, \cdots, z_{n_{i}}^{i}\right\}, i=1, \cdots, k$ be a set of regular sub-basis of $\left\{x_{1}, \cdots, x_{n}\right\}$. Assume

$$
\left\{z_{1}^{i}, \cdots, z_{n_{i}}^{i}\right\} \subset F_{\ell}\left\{z_{1}^{i+1}, \cdots, z_{n_{i+1}}^{i+1}\right\}, \quad i=1, \cdots, k-1 .
$$

Then there exists a coordinate frame $w_{1}, \cdots, w_{n}$, such that

$$
F_{\ell}\left\{z_{1}^{i}, \cdots, z_{n_{i}}^{i}\right\}=F_{\ell}\left\{w_{1}, \cdots, w_{n_{i}}\right\}, \quad i=1, \cdots, k .
$$

Corollary 15. Let $Y$ and $Z$ be two regular subspaces and $Y \subset Z$. Then there exists a regular subspace $W$ such that $F_{\ell}(W, Y)=Z$, which is denoted by

$$
W \oplus Y=Z \text {. }
$$

Remark 16. If (31) holds, $W$ is called the complement of $Y$ in $Z$, denoted by $W=Z \backslash Y$. It is obvious that $W$ is not unique.

\section{State Space Coordinate Transformation}

This section considers the logical coordinate transformation of Boolean (control) networks.

Consider the dynamics of a Boolean network (without control). Assume that its algebraic form is

$$
x(t+1)=L x(t)
$$

Let $z=T x$ be a logical coordinate change. Then

$$
z(t+1)=T x(t+1)=T L x(t)=T L T^{-1} z(t) .
$$

That is, the dynamics of the Boolean network (32) becomes

$$
z(t+1)=T L T^{T} z(t)
$$

In fact, this is similar to any discrete-time linear dynamic systems.

Next, we consider the Boolean control system (2). Denote its algebraic form as

$$
\left\{\begin{array}{l}
x(t+1)=L u(t) x(t) \\
y(t)=H x(t)
\end{array}\right.
$$

Then

$$
\begin{aligned}
z(t+1) & =T x_{t+1}=T L u(t) x(t)=T L u(t) T^{T} z(t) \\
& =T L\left(I_{2^{m}} \otimes T^{T}\right) u(t) z(t) .
\end{aligned}
$$


This form with a similar computation for $y$ shows that under the state space coordinate transformation $z=T x$ system (34) can be expressed as

$$
\left\{\begin{array}{l}
z(t+1)=\tilde{L} u(t) z(t), z \in \Delta_{2^{n}} \\
y(t)=\tilde{H} z(t), y \in \Delta_{2^{p}},
\end{array}\right.
$$

where

$$
\tilde{L}=T L\left(I_{2^{m}} \otimes T^{T}\right) ; \quad \tilde{H}=H T^{T}
$$

(36) is very useful in our further investigation.

We give an example to describe this.

Example 17. Consider the following system

$$
\left\{\begin{array}{l}
x_{1}(t+1)=\neg\left(x_{1}(t) \leftrightarrow x_{2}(t)\right) \\
x_{2}(t+1)=\neg\left(x_{2}(t) \leftrightarrow x_{3}(t)\right) \\
x_{3}(t+1)=u(t) \wedge x_{1}(t), \\
y(t)=x_{1}(t) \leftrightarrow x_{2}(t) .
\end{array}\right.
$$

In algebraic form, it becomes

$$
\left\{\begin{array}{l}
x_{1}(t+1)=M_{p} x_{1} x_{2}(t) \\
x_{2}(t+1)=M_{p} x_{2}(t) x_{3}(t) \\
x_{3}(t+1)=M_{c} u(t) x_{1}(t), \\
y(t)=M_{e} x_{1}(t) x_{2}(t) .
\end{array}\right.
$$

Let $x(t)=x_{1}(t) x_{2}(t) x_{3}(t)$. Then

$$
x(t+1)=M_{p} x_{1} x_{2} M_{p} x_{2} x_{3} M_{c} u x_{1}:=L u(t) x(t),
$$

where $L \in M_{8 \times 16}$ can be easily calculated as

$$
\begin{aligned}
L & =M_{p}\left(I_{4} \otimes M_{p}\right)\left(I_{2} \otimes M_{r}\right)\left(I_{8} \otimes M_{c}\right) W_{[4,8]}\left(I_{2} \otimes M_{r}\right) \\
& =\delta_{8}[7,5,1,3,4,2,6,8,8,6,2,4,4,2,6,8] .
\end{aligned}
$$

Since there is no $x_{3}$ in $y$, we introduce a dummy matrix, as $E_{d}=\delta_{2}\left[\begin{array}{lll}1 & 1 & 2\end{array}\right]$, then we have (Cheng and Qi, 2009b)

$$
E_{d} p q=p
$$

Using it, $y$ can be expressed as

$$
\begin{aligned}
y(t) & =M_{e} x_{1}(t) E_{d} x_{2}(t) x_{3}(t) \\
& =M_{e}\left(I_{2} \otimes E_{d}\right) x(t) \\
& =\delta_{2}\left[\begin{array}{llllllll}
1 & 2 & 1 & 2 & 2 & 1 & 2 & 1
\end{array}\right] x(t) .
\end{aligned}
$$

Assume that we use the coordinate change $z=T x$ as

$$
\left\{\begin{array}{l}
z_{1}=x_{1} \bar{\nabla} x_{2} \\
z_{2}=\neg x_{1} \\
z_{3}=\neg x_{3}
\end{array}\right.
$$

which is the inverse of coordinate change in (16). So its transfer matrix is $T^{T}$, where $T$ is as in (19).

Using logical coordinate transformation (35), we have

$$
\begin{aligned}
& \tilde{L}=T L\left(I_{2} \otimes T^{T}\right) \\
& =\delta_{8}[7,3,4,8,5,1,2,6,7,3,3,7,5,1,1,5] \text {; } \\
& \tilde{H}=H T^{T}=\delta_{2}\left[\begin{array}{lllllll}
1 & 2 & 2 & 1 & 1 & 2 & 2
\end{array}\right] \text {. }
\end{aligned}
$$

We also have

$$
\begin{aligned}
& \tilde{M}_{1}=S_{1}^{3} \tilde{L}=\delta_{2}[2112211221122112] \text {; } \\
& \tilde{M}_{2}=S_{2}^{3} \tilde{L}=\delta_{2}[2222111122221111] \text {; } \\
& \tilde{M}_{3}=S_{3}^{3} \tilde{L}=\delta_{2}\left[\begin{array}{llll}
1 & 122112211111 & 1 & 11
\end{array}\right] \text {. }
\end{aligned}
$$

Using the converting procedure,

$$
\begin{aligned}
z_{1}(t+1)= & {\left[u(t) \wedge g_{1}^{1}\left(x_{1}(t), x_{2}(t), x_{3}(t)\right)\right] \vee } \\
& {\left[\neg u(t) \wedge g_{1}^{2}\left(x_{1}(t), x_{2}(t), x_{3}(t)\right)\right] . }
\end{aligned}
$$

Since $\tilde{M}_{1}^{1}=\tilde{M}_{1}^{2}$, then $g_{1}^{1}=g_{1}^{2}$, we conclude that

$$
z_{1}(t+1)=g_{1}^{1}\left(x_{1}(t), x_{2}(t), x_{3}(t)\right) .
$$

Continuing this mechanical process, we finally get the expression of the Boolean control network (37) under $z$ coordinate frame as

$$
\left\{\begin{array}{l}
z_{1}(t+1)=z_{2}(t) \bar{\vee} z_{3}(t) \\
z_{2}(t+1)=\neg z_{1}(t) \\
z_{3}(t+1)=u(t) \rightarrow z_{2}(t) \\
y(t)=z_{2}(t) \leftrightarrow z_{3}(t)
\end{array}\right.
$$

\section{Decomposition and Normal Forms}

First, we introduce the incidence matrix of a logical mapping (Robert, 1986).

Consider a logical mapping $F: \mathcal{D}^{n} \rightarrow \mathcal{D}^{m}$, described as

$$
F: y_{i}=f_{i}\left(x_{1}, \cdots, x_{n}\right), \quad i=1, \cdots, m
$$

$f_{i}$ is said to be a clear form, if $f_{i}$ has no fabricated arguments. That is, if $f_{i}$ is independent of $x_{j}$ then $x_{j}$ will not appear into $f_{i}$. Note that in a logical function, it is not obvious to identify if an argument is fabricated or not. Cheng and Qi (2009a) provided a mechanical procedure to get the clear form of arbitrary logical function $f$. Hereafter we assume that the logical equations concerned are in clear form. That is, there are no fabricated variables. 
For mapping $F$ with clear $f_{i}$, its incidence matrix is an $m \times n$ matrix $B(F)$, whose entries are defined by

$$
b_{i, j}= \begin{cases}1, & \text { if } x_{j} \text { appears into } f_{i} \\ 0, & \text { otherwise }\end{cases}
$$

Recall system (2). Denote the incidence matrices for mapping $F$ with respect to $x, u$ and the mapping $H$ with respect to $x$ respectively by $B(F) \in M_{n, n+m}$, and $B(H) \in M_{p, m}$. For convenience, we arrange $B(F)$ in such a way: the first $n$ columns are for $x$ and the last $m$ columns are for $u$. That is, $b_{i, j}=1, j \leq n$ means $x_{j}$ appears in $f_{i}(x, u)$ and $b_{i, j}=1, j>n$ means $u_{j-n}$ appears in $f_{i}(x, u)$.

Definition 18. (1) A subspace $V=F_{\ell}\left\{x_{j_{1}}, x_{j_{2}}, \cdots, x_{j_{\beta}}\right\}$ is called an uncontrollable subspace, if it does not affected by $\{u(t)\}$.

(2) A subspace $V=F_{\ell}\left\{x_{k_{1}}, x_{k_{2}}, \cdots, x_{k_{\gamma}}\right\}$ is said to be an unobservable subspace if the output $y_{j}(t), j=$ $1,2, \cdots, p$ are not affected by $x_{k_{\ell}}(t), \ell=1,2, \cdots, \gamma$ under arbitrary controls $\{u(t)\}$.

To make the definition more clear, we consider the incidence matrices. If (after possible variable reordering) the incidence matrix of state equations becomes

$$
B(F)=\left[\begin{array}{ccc}
L_{11} & L_{12} & L_{13} \\
0 & L_{22} & 0
\end{array}\right]
$$

where the first block row corresponds to equations of $x^{1}=\left(x_{1}, \cdots, x_{k}\right)$, and the second to $x^{2}=$ $\left(x_{k+1}, \cdots, x_{n}\right)$; the first, second and third block columns correspond to $x^{1}, x^{2}$ and $u$ respectively. Then $x^{2}=\left(x_{k+1}, \cdots, x_{n}\right)$ is an uncontrollable subspace.

Similarly, if (after possible variable reordering) the incidence matrix of states becomes

$$
B(F)=\left[\begin{array}{ccc}
L_{11} & 0 & L_{13} \\
L_{21} & L_{22} & L_{23}
\end{array}\right]
$$

and the incidence matrix of outputs becomes

$$
B(H)=\left[\begin{array}{ll}
H_{11} & 0
\end{array}\right]
$$

where the block decompositions of $B(F)$ and $B(H)$ are corresponding to the block coordinates $x^{1}=$ $\left(x_{1}, \cdots, x_{k^{\prime}}\right)$, and $x^{2}=\left(x_{k^{\prime}+1}, \cdots, x_{n}\right)$ (and $u$ for last block column $B(F))$. Then $x^{2}=\left(x_{k^{\prime}+1}, \cdots, x_{n}\right)$ is the unobservable subspace.

Unfortunately, the aforementioned definition is coordinatedepending. It may be seen from the following example.
Example 19. Consider the following system

$$
\left\{\begin{aligned}
& x_{1}(t+1)=\left(u(t) \wedge\left(x_{1}(t) \vee x_{2}(t)\right)\right) \vee \\
&\left(\neg u(t) \wedge\left(x_{1}(t) \wedge x_{2}(t)\right)\right) \\
& x_{2}(t+1)= x_{1}(t) \wedge x_{2}(t) \\
& y(t)=x_{2}(t) .
\end{aligned}\right.
$$

We have the incidence matrices as

$$
B(F)=\left[\begin{array}{lll}
1 & 1 & 1 \\
1 & 1 & 0
\end{array}\right]
$$

and

$$
B(H)=\left[\begin{array}{ll}
0 & 1
\end{array}\right]
$$

It is easy to check that even with reordering the variables we can get neither uncontrollable nor unobservable subspace. Skipping a normal routine computation, we give the algebraic form of the system (45):

$$
\left\{\begin{array}{l}
x(t+1)=L u(t) x(t) \\
y=H x(t)
\end{array}\right.
$$

where

$$
L=\delta_{8}[1,2,2,4,1,4,4,4] ; \quad H=\delta_{2}[1,2,1,2] .
$$

Now we consider a coordinate transformation:

$$
\left\{\begin{array}{l}
z_{1}=x_{1} \bar{\vee} x_{2} \\
z_{2}=\neg x_{2}
\end{array}\right.
$$

Note that since

$$
\begin{aligned}
z & =M_{p} x_{1} x_{2} M_{n} x_{2}=M_{p}\left(I_{4} \otimes M_{n}\right) x_{1} M_{r} x_{2} \\
& =M_{p}\left(I_{4} \otimes M_{n}\right)\left(I_{2} \otimes M_{r}\right) x:=T x,
\end{aligned}
$$

then

$$
L=M_{p}\left(I_{4} \otimes M_{n}\right)\left(I_{2} \otimes M_{r}\right)=\delta_{4}[4,1,2,3],
$$

which is nonsingular. Hence $z=T x$ is a coordinate transformation.

Under coordinate frame $z$, we have

$$
\left\{\begin{array}{l}
z(t+1)=\tilde{L} u(t) z(t) \\
y(t)=\tilde{H} z(t),
\end{array}\right.
$$

where, by using (36),

$$
\begin{gathered}
\tilde{L}=T L\left(I_{2} \otimes T^{T}\right)=\delta_{8}[1,1,3,4,3,3,3,4] ; \\
\tilde{H}=\delta_{2}[2,1,2,1] .
\end{gathered}
$$


Using the standard procedure provided in Cheng and Qi (2009a), we can reconstruct the dynamics of (47). Skipping the routine computation, we have

$$
\left\{\begin{array}{l}
z_{1}(t+1)=z_{1}(t) \wedge u \\
z_{2}(t+1)=z_{1}(t) \vee z_{2}(t) \\
y(t)=\neg z_{1}(t)
\end{array}\right.
$$

Observing (48), one sees easily that $z_{2}$ is unobservable subspace.

We give the following coordinate free definition.

Definition 20. (1) Let $\xi(x) \in \mathcal{U}$, with structure matrix $M_{\xi} \cdot \xi$ is said to be uncontrollable if

$$
\xi(t+1)=M_{\xi} x(t+1)=M_{\xi} L x(t) u(t)
$$

is $u(t)$-independent, i.e., it does not affected by $u(t)$. Let $\mathcal{C}_{c} \subset \mathcal{U}$ be the subspace of all $u(t)$-independent functions, called the largest uncontrollable subspace.

(2) A regular subspace $Z_{r}$ is called an unobservable subspace, if under the coordinate $Z=\left\{Z_{r}, Z_{r}^{c}\right\}$, it is unobservable. Let $\mathcal{O}_{c}$ be the set of functions of all unobservable regular subspaces, i.e., $\mathcal{O}_{c}=$ $F_{\ell}\left\{Z_{r} \mid Z_{r}\right.$ is unobservable regular subspaces $\}$, called the largest unobservable subspace.

The following two proposition about controllable and observable normal forms respectively is an immediate consequence of the definition.

Proposition 21. (1) Assume that the largest uncontrollable subspace $\mathcal{C}_{c}$ is a regular subspace, then there exists a state space expression of (2) which have largest uncontrollable subspace $z^{2}$ (unique up to a coordinate transformation) as

$$
\left\{\begin{array}{l}
z^{1}(t+1)=F^{1}(z(t), u(t)) \\
z^{2}(t+1)=F^{2}\left(z^{2}(t)\right)
\end{array}\right.
$$

(49) will be called the normal controllable form.

(2) Assume that the unobservable subspace $\mathcal{O}_{c}$ is a regular subspace, then there exists an expression of (2) which have largest unobservable subspace $z^{2}$ (unique up to a coordinate transformation) as

$$
\left\{\begin{array}{l}
z^{1}(t+1)=F^{1}\left(z^{1}(t), u(t)\right), \\
z^{2}(t+1)=F^{2}(z(t), u(t)) ; \\
y(t)=H\left(z^{1}(t)\right) .
\end{array}\right.
$$

(50) will be called the normal observable form.

Finally, we propose a Kalman decomposition form. Consider system (2). Assume that $\mathcal{C}_{c}, \mathcal{O}_{c}, \mathcal{C}_{c} \cup \mathcal{O}_{c}$, and $\mathcal{C}_{c} \cap \mathcal{O}_{c}$ are regular subspaces of $\{x\}$. Denote

$$
\begin{aligned}
& V_{1}=\mathcal{C} \cap \mathcal{O}:=D^{n} \backslash\left(\mathcal{C}_{c} \cup \mathcal{O}_{c}\right), V_{2}=\mathcal{C} \cap \mathcal{O}_{c}:=\left(\mathcal{C}_{c} \cup \mathcal{O}_{c}\right) \backslash \mathcal{C}_{c} \\
& V_{3}=\mathcal{C}_{c} \cap \mathcal{O}:=\mathcal{C}_{c} \backslash\left(\mathcal{C}_{c} \cap \mathcal{O}_{c}\right), V_{4}=\mathcal{C}_{c} \cap \mathcal{O}_{c} .
\end{aligned}
$$

Theorem 22. Assume that $\mathcal{C}_{c}, \mathcal{O}_{c}, \mathcal{C}_{c} \cup \mathcal{O}_{c}$, and $\mathcal{C}_{c} \cap \mathcal{O}_{c}$ are regular subspaces of $\{x\}$. System (2) has the following Kalman decomposition:

$$
\begin{aligned}
& \left\{\begin{array}{l}
z^{1}(t+1)=F^{1}\left(z^{1}(t), z^{3}(t), u(t)\right), \quad z^{1} \in V_{1} \\
z^{2}(t+1)=F^{2}\left(z^{1}(t), z^{2}(t), z^{3}(t), z^{4}(t), u(t)\right), z^{2} \in V_{2} \\
z^{3}(t+1)=F^{3}\left(z^{3}(t)\right), \quad z^{3} \in V_{3} \\
z^{4}(t+1)=F^{4}\left(z^{3}(t), z^{4}(t)\right), \quad z^{4} \in V_{4} ;
\end{array}\right. \\
& y_{s}(t)=h_{s}\left(z^{1}(t), z^{3}(t)\right), \quad s=1,2, \cdots, p,
\end{aligned}
$$

where $z^{1}(t)$ is the controllable and observable subspace, $z^{2}(t)$ is the controllable and unobservable subspace, $z^{3}(t)$ is the uncontrollable and observable subspace, and $z^{4}(t)$ is the uncontrollable and unobservable subspace. Moreover, the expression is unique up to a coordinate transformation.

PROOF. Consider the nested regular subspaces

$$
\mathcal{C}_{c} \cup \mathcal{O}_{c} \supset \mathcal{C}_{c} \supset \mathcal{C}_{c} \cap \mathcal{O}_{c}
$$

Assume $\operatorname{dim} V_{i}=n_{i}, i=1,2,3,4$. Denote $j_{1}=n_{1}$, $j_{2}=n_{1}+n_{2}, j_{3}=n_{1}+n_{2}+n_{3}$. According to Corollary 14 , we can find a coordinate frame $z=\left\{z_{i} \mid i=1, \cdots, n\right\}$, such that

$$
\begin{aligned}
& \mathcal{C}_{c} \cup \mathcal{O}_{c}=F_{\ell}\left\{z_{k} \mid k>j_{1}\right\} ; \\
& \mathcal{C}_{c}=F_{\ell}\left\{z_{k} \mid k>j_{2}\right\} ; \\
& \mathcal{C}_{c} \cap \mathcal{O}_{c}=F_{\ell}\left\{z_{k} \mid k>j_{3}\right\} .
\end{aligned}
$$

Under this coordinate frame (51) follows immediately.

Next, we give an example to depict this. 
Example 23. Consider the following system

$$
\left\{\begin{aligned}
& x_{1}(t+1)= u \\
& x_{2}(t+1)= \neg x_{2}(t) \\
& x_{3}(t+1)= {\left[x_{3}(t) \wedge x_{4}(t) \wedge\left(x_{5}(t) \leftrightarrow x_{6}(t)\right)\right] \vee } \\
& {\left[x_{3}(t) \wedge\left(\neg\left(x_{4}(t)\right) \wedge x_{5}(t)\right] \vee\left(\neg x_{3}(t)\right)\right.} \\
& x_{4}(t+1)= \neg\left(x_{1}(t) \leftrightarrow x_{2}(t)\right) \\
& x_{5}(t+1)= {\left[x_{1}(t) \wedge\left(x_{2}(t) \leftrightarrow x_{3}(t)\right)\right] \vee\left[\left(\neg x_{1}(t)\right) \wedge\right.} \\
&\left.\left(\neg\left(x_{2}(t) \leftrightarrow x_{3}(t)\right)\right)\right] \\
& x_{6}(t+1)= {\left[x_{1}(t) \leftrightarrow x_{2}(t)\right] \wedge\left\{\left[x_{4}(t) \wedge\right.\right.} \\
&\left.\left.\left(x_{5}(t) \leftrightarrow x_{6}(t)\right)\right] \vee\left[\left(\neg x_{4}(t)\right) \wedge x_{5}(t)\right]\right\}, \\
& y_{1}(t)=\neg x_{4}(t), \\
& y_{2}(t)=\left(x_{1}(t) \leftrightarrow x_{2}(t)\right) \rightarrow\left(\neg x_{2}(t)\right) .
\end{aligned}\right.
$$

We skip the tedious process for finding the subspaces by using coordinate transformations, and give the logical coordinate transformation as follows:

$$
\left\{\begin{array}{l}
z_{1}(t)=x_{1}(t) \leftrightarrow x_{2}(t) \\
z_{2}(t)=x_{4}(t) \\
z_{3}(t)=x_{6}(t) \\
z_{4}(t)=\neg x_{2}(t) \\
z_{5}(t)=\neg x_{3}(t) \\
z_{6}(t)=\left[x_{4}(t) \wedge\left(x_{5}(t) \leftrightarrow x_{6}(t)\right)\right] \vee\left[\left(\neg x_{4}(t)\right) \wedge x_{5}(t)\right]
\end{array}\right.
$$

Its inverse mapping is:

$$
\left\{\begin{array}{l}
x_{1}(t)=\neg\left(z_{1}(t) \leftrightarrow z_{4}(t)\right) \\
x_{2}(t)=\neg z_{4}(t) \\
x_{3}(t)=z_{5}(t) \\
x_{4}(t)=z_{2}(t) \\
x_{5}(t)=\left[z_{2}(t) \wedge\left(z_{3}(t) \leftrightarrow z_{6}(t)\right)\right] \vee\left[\left(\neg z_{2}(t)\right) \wedge z_{6}(t)\right] \\
x_{6}(t)=z_{3}(t)
\end{array}\right.
$$

Using (53)-(54), it is easy to calculate that under $\left\{z_{i}\right\}$ coordinate frame system (52) can be converted into the following form:

$$
\begin{gathered}
\left\{\begin{array}{l}
z_{1}(t+1)=z_{4}(t) \leftrightarrow u \\
z_{2}(t+1)=\neg z_{1}(t) \\
z_{3}(t+1)=z_{1}(t) \wedge z_{6}(t) \\
z_{4}(t+1)=\neg z_{4}(t) \\
z_{5}(t+1)=z_{5}(t) \vee z_{6}(t) \\
z_{6}(t+1)=\neg z_{5}(t)
\end{array}\right. \\
y_{1}(t)=\neg z_{2}(t) \\
y_{2}(t)=z_{1}(t) \rightarrow z_{4}(t) .
\end{gathered}
$$

It is easy to check that (55) is the Kalman decomposition form of system (52) with

$$
\begin{gathered}
\mathcal{C} \cap \mathcal{O}=F_{\ell}\left\{z_{1}(t), z_{2}(t)\right\} ; \quad \mathcal{C} \cap \mathcal{O}_{c}=F_{\ell}\left\{z_{3}(t)\right\} \\
\mathcal{C}_{c} \cap \mathcal{O}=F_{\ell}\left\{z_{4}(t)\right\} ; \quad \mathcal{C}_{c} \cap \mathcal{O}_{c}=F_{\ell}\left\{z_{5}(t), z_{6}(t)\right\}
\end{gathered}
$$

\section{Realization}

Definition 24. Given two Boolean control networks. They are said to be equivalent if for any point $x_{0}$ of one network there is a point $\tilde{x}_{0}$ of the other network such that for the same inputs $u(t), t=0,1,2, \cdots$ with initial values $x_{0}$ and $\tilde{x}_{0}$ respectively, the outputs $\{y(t)\}$ are the same.

Consider a linear control system (Wonham, 1979)

$$
\begin{cases}\dot{x}=A x+B u, & x \in \mathbb{R}^{n}, u \in \mathbb{R}^{m} \\ y=C x, & y \in \mathbb{R}^{p} .\end{cases}
$$

Its Kalman decomposition form is

$$
\left[\begin{array}{c}
\dot{z}^{1} \\
\dot{z}^{2} \\
\dot{z}^{3} \\
\dot{z}^{4}
\end{array}\right]=\left[\begin{array}{cccc}
A_{11} & 0 & A_{13} & 0 \\
A_{21} & A_{22} & A_{23} & A_{24} \\
0 & 0 & A_{33} & 0 \\
0 & 0 & A_{33} & A_{34}
\end{array}\right]\left[\begin{array}{c}
z^{1} \\
z^{2} \\
z^{3} \\
z^{4}
\end{array}\right]
$$

$$
y(t)=\left[\begin{array}{llll}
C_{1} & 0 & C_{3} & 0
\end{array}\right] z .
$$

Then its minimum realization is

$$
\left\{\begin{array}{l}
\dot{x}^{1}=A_{11} x^{1} \\
y=C_{1} x^{1}
\end{array}\right.
$$

We define the minimum realization of system (2) in a mimic way.

Definition 25. Consider system (2) with its Kalman decomposition (51). Given a fixed (frozen) value $z^{3}=$ $z_{0}^{3}$, the minimum realization of system (2) with frozen $z^{3}=z_{0}^{3}$ is defined by

$$
\left\{\begin{array}{l}
z^{1}(t+1)=F^{1}\left(z^{1}(t), A_{3}^{t} z_{0}^{3}, u(t)\right) \\
y_{s}(t)=h_{s}\left(z^{1}(t), A_{3}^{t} z_{0}^{3}\right), \quad s=1,2, \cdots, p
\end{array}\right.
$$

where $A_{3}$, as the structure matrix of $F^{3}$, is an $n_{3} \times n_{3}$ known logical matrix, and $z_{0}^{3}$ is a parameter, which is adjustable. 
Note that in general the minimum realization depends on $A_{3}$ and $z_{0}^{3}$. In the following two cases the minimum realization is unique:

- Case 1. $z^{3}$ does not appear into the dynamic equation of $z^{1}$.

- Case 2. subsystem of $z^{3}$ globally converges to $\xi$. Then in (59) we can replace $A_{3}^{t} z_{0}^{3}$ by $\xi$, and call (59) the stationary state realization.

Example 26. Recall Example 23. To get the minimum realization of $(52)$, we write the first block equation by using its Kalman decomposition form (55).

$$
\left\{\begin{array}{l}
z_{1}(t+1)=z_{4}(t) \leftrightarrow u \\
y_{1}(t)=\neg z_{4}(t), \\
y_{2}(t)=z_{1}(t) \rightarrow z_{4}(t) .
\end{array}\right.
$$

Note that in (55) the third block variable is $z^{3}=z_{4}$. Since $z_{4}=M_{n}^{t} z_{4}^{0}$, we have the minimum realization as

$$
\left\{\begin{array}{l}
z_{1}(t+1)=M_{e} M_{n}^{t} z_{4}^{0} u \\
y_{1}(t)=M_{n}^{t+1} z_{4}^{0}, \\
y_{2}(t)=M_{i} z_{1}(t) M_{n}^{t} z_{4}^{0} .
\end{array}\right.
$$

It is easy to verify that the input-output mapping of system $(52)$ with initial value $\left(z_{1}^{0}, \cdots, z_{6}^{0}\right)$ is exactly the same as (61) with initial value $z_{1}^{0}$ and parameter $z_{4}^{0}$.

\section{Conclusion}

In this paper we consider the realization problem of Boolean control systems. First, we give a rigorous definition for the coordinate transformation of the state variables of a Boolean network. Then the coefficient matrices of the Boolean (control) systems under algebraic expression are investigated. The formulas are obtained for system coefficients under coordinate transformation. Introducing the concept of regular sub-basis and regular subspace, we then investigate the controllable and observable normal forms. Under certain regularity assumption, the Kalman decomposition of Boolean control networks is presented. Finally, based on the Kalman decomposition the minimum realization is obtained.

\section{References}

Akutsu, T., Hayashida, M., Ching, W., Ng, M. K., 2007. Control of Boolean networks: hardness results and algorithms for tree structured networks. J. Theoretical Biology 244, 670-679.

Albert, R., Othmer, H. G., 2003. The topology and signature of the regulatory interactions predict the expression pattern of the segment polarity genes in Drospphila melanogaster. J. Theor. Bio. 223 (1), 1-18.
Cheng, D., 2007. Semi-tensor product of matrices and its applications - A survey. In: Proc. ICCM 2007. Vol. 3. pp. 641-668.

Cheng, D., March 2009. Input-state approach to Boolean networks. IEEE Trans. Neural Networks 20 (3), 512521.

Cheng, D., Qi, H., 2009a. Controllability and observability of Boolean control networks. Automatica 45 (7), 1659-1667.

Cheng, D., Qi, H., 2009b. A linear representation of dynamics of Boolean networks, provisionally accepted by IEEE Trans. Aut. Contr. (Preprint: http://lsc.amss.ac.cn/ dcheng/preprint).

Davidson, E., Rast, J., Oliveri, P., Ransick, A., Calestani, C., Yuh, C., Minokawa, T., Amore, G., Hinman, V., Arenas-Mena, C., et al., 2002. A genomic regulatory network for development. Science 295 (5560), 1669-1678.

Farrow, C., Heidel, J., Maloney, H., Rogers, J., 2004. Scalar equations for synchronous Boolean networks with biological applications. IEEE Trans. Neural Networks 15 (2), 348-354.

Huang, S., 2002. Regulation of cellular states in mammalian cells from a genomewide view. In: Julio, C.-V., Hofestadt, R. (Eds.), Gene Regulation and Metabolism. MIT Press, Cambridge, MA, pp. 181220.

Huang, S., Ingber, I., 2000. Shape-dependent control of cell growth, differentiation, and apotosis: Switching between attractors in cell regulatory networks. Exp. Cell Res. 261, 91-103.

Kauffman, S. A., 1969. Metabolic stability and epigenesis in randomly constructed genetic nets. J. Theoretical Biology 22, 437-467.

Rade, L., Westergren, B., 1998. Mathematics Handbook for Science and Engineering, 4th Edition. Studentlitteatur, Sweden.

Robert, F., 1986. Discrete Iterations: A Metric Study. Springer-Verlag, Berlin, translated by J. Rokne.

Wonham, W., 1979. Linear Multivariable Control: A Geometric Approach, 2nd Edition. Springer, Berlin. 